\title{
Efectos del COVID-19 sobre los ingresos y determinación de brechas sociales en Tacna
}

\author{
Effects of COVID-19 on income and determination of social gaps in Tacna
}

$\begin{array}{r}\text { Irene Limache-Ortiz } \\ \text { Universidad Nacional Jorge Basadre Grohmann, Tacna-Perú. } \\ \text { ORCID: 0000-0002-5024-2775 } \\ \text { ilimacheo@unjbg,edu.pe } \\ \hline\end{array}$

\section{RESUMEN}

El presente estudio tuvo como finalidad determinar el impacto del COVID-19 en el aporte de los ingresos promedio de los trabajadores del sector primario, secundario y terciario en la región de Tacna y su efecto en la polarización social, así como establecer el impacto del COVID-19 en la propensión al ahorro mensual. El tipo de investigación fue descriptivo, el instrumento de medición utilizado fue el cuestionario, mediante la aplicación de la encuesta. Se determinó un decremento de los ingresos económicos promedios que asciende a $-55.6 \%,-50.0 \%$ y $-85.0 \%$ para los sectores primario, secundario y terciario respectivamente, así como la propensión marginal al ahorro disminuyó en $-73.4 \%$ debido al COVID-19 en la región de Tacna. La disminución de los ingresos promedio de los trabajadores origina una disminución en el consumo y este a su vez genera depresión económica que conlleva inicialmente a una recesión y según la dinámica del flujo de dinero que el estado despliegue existe una alta probabilidad que se desencadene una inflación.

Palabras clave: Ahorro, COVID-19, ingresos.

\begin{abstract}
The purpose of this study was to determine the impact of COVID-19 on the average income contribution of workers in the primary, secondary and tertiary sectors in the Tacna region and its effect on social polarization, as well as to establish the impact of COVID-19 on the propensity to save on a monthly basis. The type of research was descriptive, the measurement instrument used was the questionnaire, through the application of the survey. It was determined that the average economic income was determined that amounted to $-55.6 \%,-50.0 \%$ and $-85.0 \%$ for the primary, secondary and tertiary sectors respectively, as well as the marginal propensity to save decreased by $-73.4 \%$ due to COVID-19 in the Tacna region. The decline in the average income of workers leads to a decrease in consumption, which in turn generates economic depression that initially leads to a recession. According to the dynamics of the flow of money that the state deploys, there is a high probability that inflation will be triggered.
\end{abstract}

Keywords: Saving, COVID-19, income.

\section{INTRODUCCIÓN}

Según After the Pandemic (2020), el COVID-19 está generando un gran impacto negativo a corto plazo en el crecimiento económico mundial, reflejado en uno de los indicadores macroeconómicos más relevantes del país como es el producto bruto interno (PBI); teniendo como resultado que, hasta lo que va del año, el PBI peruano cayera en $30.2 \%$ en el segundo trimestre. Mientras que para Araújo (2020), dada la situación de pandemia a nivel mundial, el gobierno peruano se ha visto obligado a adoptar la decisión de poner en cuarentena al país y confinar a la población. 
Considerando los limitados medios e infraestructuras, se ha propiciado que la crisis sanitaria y sus consecuencias económicas resulten ser desiguales y afecten con mayor impacto a las poblaciones más pobres (Paiva et al., 2020). Lo cual genera una creciente preocupación y motiva la realización del presente artículo para determinar los efectos del COVID-19 sobre los ingresos y brechas sociales en Tacna, centrándose específicamente en el segundo trimestre del presente año.

Respecto a la disminución de los ingresos promedios, producto de la pérdida de empleo o paralización de las actividades en los sectores económicos primario, secundario, terciario o de servicios y sus subsectores, enmarcados en una lógica comparativa entre las diferentes clases socioeconómicas, como resultado del análisis se determinó que de los trabajadores del sector primario, secundario y terciario en la región de Tacna, se vieron perjudicados con un decremento significativo que asciende a $-55.6 \%,-50.0 \%$ y $-85.0 \%$ respectivamente. Asimismo, la propensión marginal al ahorro disminuyó en $-73.4 \%$ debido al COVID-19, cuyo resultado confirma la relación directa que existe entre las variables de los ingresos y la propensión al ahorro (Nava \& Brown, 2018). Situación que pone en alerta no solo al Estado, sino al aparato productivo de este sector, para la búsqueda de alternativas de solución; dentro de ellas, la utilización intensiva de tecnología que ayude a su recuperación. Esta alternativa puede generar controversias, teniendo en cuenta que existe una alta probabilidad del desplazamiento de mano de obra.

Entonces, es oportuno que el Estado dentro de su manejo keynesiano apueste por esta mano de obra desplazada y la oriente hacia su desarrollo, teniendo como prioridad el adiestramiento en el manejo de tecnologías y la valoración de la creatividad.

\section{OBJETIVOS}

Determinar los efectos del COVID-19 sobre los ingresos y brechas sociales en Tacna.

\section{Objetivos específicos}

1) Determinar los efectos del COVID-19 sobre los ingresos y brechas sociales del sector primario y sus subsectores en Tacna.

2) Precisar los efectos del COVID-19 sobre los ingresos y brechas sociales del sector secundario y sus subsectores en Tacna.

3) Definir los efectos del COVID-19 sobre los ingresos y brechas sociales del sector terciario y sus subsectores en Tacna.

4) Determinar el aporte de los ingresos de cada sector de la economía en la región de Tacna en el contexto antes de la pandemia y en la actualidad.

5) Establecer el impacto del COVID-19 en la propensión al ahorro mensual de los pobladores de la región de Tacna.

Según After the Pandemic (2020), el COVID-19 viene generando un gran impacto negativo a corto plazo en el crecimiento económico mundial. Asimismo, las perspectivas de crecimiento a mediano plazo son más inciertas aun, encontrándose una disminución anual del PIB entre los peores vistos en los últimos 100 años. Hasta lo que va del año, el PBI peruano cayó en un $30.2 \%$ en el segundo trimestre. Concordante a ello, Siche (2020) señala que las diferentes pandemias que la humanidad ha experimentado, así como la actual enfermedad conocida como COVID- 19, tendrán un gran impacto a nivel social, económico, cultural, educativo, en el sistema de salud, en la economía, el medio ambiente y cualquier actividad humana. En la misma perspectiva, Souza (2020) evidencia que el mayor impacto negativo causado por el COVID 19 recae en el sector económico (fronteras cerradas, restaurantes cerrados, comercios cerrados) y en el sector de la salud, al ser los más afectados por la pandemia, puesto que esta crisis económica ha llevado a grandes grupos sociales a la pobreza en un corto tiempo (Berger et al., 2020).

Timmerman (1981), en la investigación Vulnerabilidad, Resiliencia y Colapso de la Sociedad, definió "Vulnerabilidad como el grado al cual un sistema, o la parte de un sistema, puede reaccionar contrario a la ocurrencia de un acontecimiento peligroso. El grado y la calidad de esa reacción adversa son condicionados en 
parte por los sistemas" (p. 30-31). Mientras que para Méndez y Prada-Trigo (2014), vulnerabilidad tiene un concepto más amplio puesto que abarca a personas, grupos sociales o territorio altamente expuestos a daños, debido a la exposición a riesgos de diversa naturaleza y escasa capacidad de respuesta y adaptación (p. 3-4).

García (2005) distingue dos conceptos importantes: la construcción social del riesgo asociada con la percepción y la construcción social del riesgo asociada la vulnerabilidad y la desigualdad, como determinantes de la magnitud de los efectos ante la presencia de la crisis económica; por lo que la vulnerabilidad se constituye en la causa principal de los procesos de desastres. Coincidentemente, Miller et al. (1967) exponen que los problemas de la pobreza, en términos de estratificación, son un problema de desigualdad. Además, es importante conocer que la pobreza definido por Sen (1992) como "ausencia de capacidades básicas no es falta de riqueza o ingreso, sino de capacidades básicas; y realiza una amplia reflexión sobre los conceptos de pobreza absoluta y relativa" (p. 5), esto se convierte en un elemento importante asociado a la vulnerabilidad.

Según Berger et al. (2020), es demasiado exigente esperar que las personas actúen en interés de la salud comunitaria a expensas de su necesidad de trabajar para mantenerse a sí mismas y a sus familias. Aunado a ello, Campos (2020) menciona que las medidas para proporcionar apoyo económico y social a la población vulnerable y a las pequeñas empresas han sido tardías y se han implementado muy lentamente con dificultades en la operacionalización.

Para Evenett (2020), la incoherencia entre las políticas comerciales nacionales y la respuesta médica amenaza la vida de las personas en el hogar y en el extranjero, puesto que la política comercial no debe utilizarse para alcanzar objetivos como aumentar la producción de suministros médicos que otros instrumentos de política pueden concretar. De acuerdo con Zimmermann et al. (2020), la crisis del coronavirus debería estimular los debates sobre el desarrollo de sistemas flexibles para ejecutar medidas apropiadas de distanciamiento entre países y determinar indicadores tempranos para rastrear potenciales pandemias futuras. Mientras que para Van Nguyen et al. (2020), las estrategias adaptadas en función del tiempo, el contexto y los recursos, así como el espíritu de mantener una alerta alta y una acción preventiva han sido importantes para frenar con éxito la propagación de la pandemia.

Según Bretas y Alon (2020), las tres estrategias de tecnología, colaboración y flexibilidad son vitales para las empresas que intentan mantenerse al día con los cambios actuales en el entorno empresarial y para hacer frente a lo que pueda venir en el futuro. Mientras que para Bonaccorsi et al. (2020), algunas consecuencias sociales y económicas de las medidas políticas adoptadas para contener la difusión de COVID-19 es que el bloqueo, que al parecer afecta de manera desigual a la fracción más pobre de la población; y la reducción en la movilidad y la conectividad inducida por este es más pronunciada para los municipios con mayor capacidad fiscal. Por ejemplo, en el Reino Unido, el gobierno por sus acciones ha comprometido entre el $20 \%$ y el $40 \%$ (las estimaciones varían) del PIB para manejar la crisis inmediata causada por el virus para apoyar al NHS y la economía, cuyos costos totales en salud, fiscal y social se mantendrán durante muchos años en el futuro (Chilton et al., 2020).

Si bien es cierto After the Pandemic (2020) pronostica que las pérdidas en la producción por la pandemia, a mediano plazo, serán moderadas; la incertidumbre de la población en torno a este pronóstico es significativa; además, se corre el riesgo que esta varíe considerablemente debido a las características diferenciadas que existen entre los países al manejar su dinámica económica. En tanto que las recesiones más prolongadas y las crisis financieras tienden a conducir a un crecimiento más débil a mediano plazo. En la certeza que todos los sectores de la economía se han visto afectados en menor o gran medida por la pandemia, se encuentra básicamente relacionada con el tipo de proceso de producción que desarrollan, considerando para ello la estructura en el sector primario, secundario y terciario (IPE, 2015), siendo el sector primario compuesto por la agricultura, caza, silvicultura y pesca; mientras que el sector secundario lo conforma la minería, manufactura y construcción; y el sector terciario o de servicios incluye actividades como comercio al por mayor y menor, transporte, hoteles, restaurantes y comunicaciones entre otros (ONU, 2015).

De acuerdo con el Instituto Nacional de Estadística e Informática - INEI (2020), se presenta la Figura 1, donde se explica la variabilidad mensual de la producción nacional en la economía peruana, la cual disminuyó en $39.93 \%$ en el mes de abril, $32.72 \%$ en mayo, $18.06 \%$ en junio, y $11.71 \%$ al mes de Julio. El $82 \%$ del resultado negativo de abril es explicado básicamente por seis sectores productivos (minería, comercio, manufactura, construcción, transporte y restaurantes). 


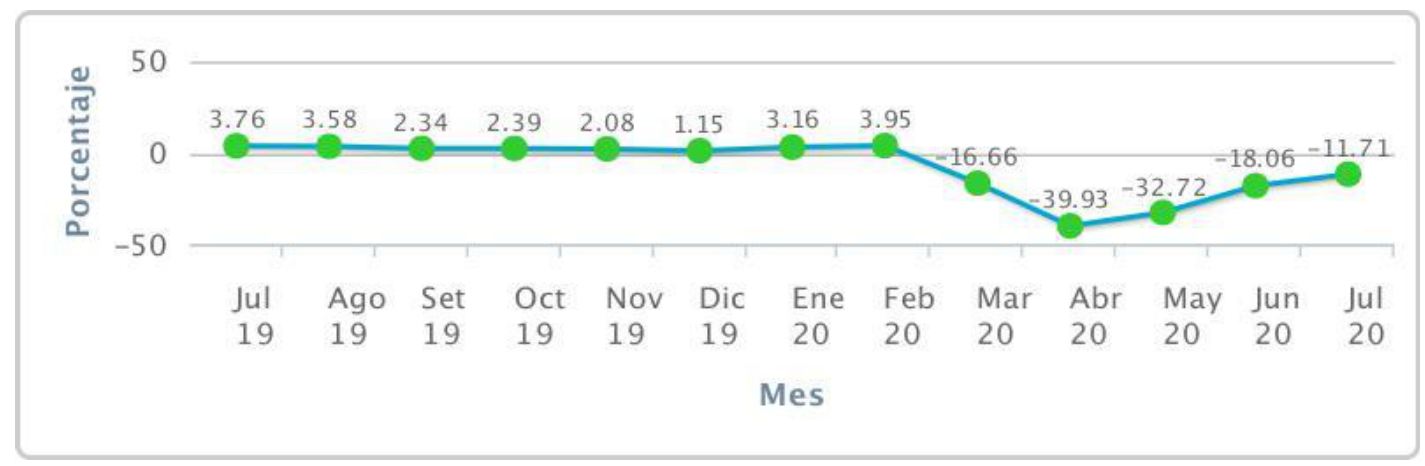

Figura1. Perú: Producción nacional Fuente: INEI 2020

El desarrollo económico se deriva del dinamismo de procesos en la estructura de producción y su contribución con el ingreso per cápita, puesto que algunos sectores económicos crecen más rápido que otros, por lo que se produce un movimiento de recursos desde la agricultura a las actividades no agrícolas seguido por un cambio desde la industria a los servicios institucionales (Fernandez, 2005). Deducimos entonces que la estructura económica de un país determina su desarrollo.

Al respecto, Kuznets (1967) resalta la propuesta de Fisher (1939) y Colin Clark (1940), quienes observaron que "el crecimiento económico, entendido como el crecimiento sostenido del ingreso per cápita, es acompañado de un proceso estructural en el cual la población ocupada se va moviendo de la agricultura hacia la industria manufacturera, y de esta a los servicios" y "durante la transición desde una economía en la que predomina el sector primario a una en la que el sector industrial y servicios se encuentren en etapas más avanzadas del desarrollo". Mientras que para Rosenstein-Rodan (1943), "la participación del sector primario, secundario y terciario y su contribución al PBI total de un país es parte del estudio del dinamismo económico" y este dinamismo económico nos abre la posibilidad de implementar una estrategia de cambios en la estructura productiva desde el sector primario y secundario hacia el sector de servicios, orientado hacia el desarrollo del país teniendo como prioridad el adiestramiento en el manejo de tecnología y la valoración de la creatividad.

El ahorro definido como el tanto por ciento del ingreso marginal que se reserva para atender las necesidades futuras, de acuerdo con lo señalado por Nava \& Brown (2018), "disminuye si el nivel de ingreso es menor al previsto y aumenta en caso contrario. La diferencia más clara entre ambas hipótesis se refiere a cómo consideran las fluctuaciones del ingreso y su influencia sobre el consumo y el ahorro" (p.18). Asimismo, varios autores concuerdan en que existe una relación positiva entre las variables ingreso y ahorro, es decir, a mayores ingresos mayor propensión marginal al ahorro.

Según Novelo (2016), en la economía keynesiana la inversión es resultado de la diferencia del ingreso y el consumo por lo que se deduce que el incremento del ingreso y la propensión al ahorro tienen una relación directamente proporcional.

\section{POBLACIÓN Y MUESTRA}

La fórmula utilizada en la presente investigación es:

Donde:

$$
\mathbf{n}=\frac{Z^{2} \cdot \text { p. q. } N}{N^{2}+Z^{2} \text { p. q. }}
$$

$\mathrm{Z}=$ Nivel de confianza

$\mathrm{N}=$ Población-Censo

$\mathrm{p}=$ Probabilidad a favor

$\mathrm{q}=$ Probabilidad en contra

$\mathrm{e}=$ error de estimación

$\mathrm{n}=$ Tamaño de muestra 
Tabla 1. Población y muestra

\begin{tabular}{cc}
\hline $\mathrm{N}$ & 187324 \\
$\mathrm{z}$ & 1.96 \\
$\mathrm{p}$ & 0.5 \\
$\mathrm{q}$ & 0.5 \\
$\mathrm{E}$ & 0.06978555 \\
\hline $\mathrm{n}$ & 197.000136 \\
\hline Fuente: Dirección de Investigación Socio Económico Laboral - MTyPE
\end{tabular}

\section{METODOLOGÍA}

El tipo de investigación es descriptiva con una muestra de 200 personas, que luego de ser depurada 1 legó a 197 individuos de la región de Tacna. El instrumento de medición utilizado fue el cuestionario validado por juicio de expertos, aplicado vía online método bola de nieve a través de Facebook, Whats app, Instagram y Twiter. Trabajo basado en primer lugar, en los datos originales producidos gracias al cuestionario elaborado en Google Formulario con un total de 19 preguntas que compartimos entre las 16:00 horas del lunes 20 y las 16:00 horas del miércoles 22 de julio. El tratamiento de datos se realizó utilizando la estadística descriptiva, específicamente distribución de frecuencias, promedios y diferenciales, además de la aplicación de una prueba estadística no paramétrica a través del Test de McNemar para observar cambios en la composición de la población con trabajo en Tacna.

\section{RESULTADOS}

De acuerdo a los objetivos planteados en el presente estudio, se determinaron los efectos del COVID-19 sobre los ingresos y brechas sociales del sector primario y sus subsectores en Tacna, de acuerdo a la Tabla 2:

\begin{tabular}{lllllll}
\multirow{2}{*}{ Tabla 2. Ingreso promedio en el sector primario } & \multicolumn{2}{c}{ Ingreso anterior } & \multicolumn{2}{l}{ Ingreso actual } & \multirow{2}{*}{ Diferencial } \\
\cline { 2 - 6 } & Rango & Absoluto & Participación & Absoluto & Participación & \\
\hline Baja & Menor a S/ 899 & 2 & $22.2 \%$ & 1 & $11.1 \%$ & $-11.1 \%$ \\
Media & $\mathrm{S} / 899-\mathrm{S} / 2192$ & 5 & $55.6 \%$ & 3 & $33.3 \%$ & $-22.2 \%$ \\
Alta & $\mathrm{S} / 2192-\mathrm{S} / 4384$ & 1 & $11.1 \%$ & 0 & $0.0 \%$ & $-11.1 \%$ \\
Más alta & $\mathrm{S} / 4384$ a más & 1 & $11.1 \%$ & 0 & $0.0 \%$ & $-11.1 \%$ \\
\hline Total & & 9 & $100 \%$ & 4 & $44.4 \$$ & $-55.6 \%$ \\
\hline
\end{tabular}

Para el sector agricultura, la situación anterior a la cuarentena presenta la siguiente distribución de ingresos promedio: el $57.1 \%$ de la población percibió ingresos entre S/ 899 y S/. 2192, el 28.6\% accedió a ingresos menores a S/ 899, el $14.3 \%$ tuvo ingresos entre S/ 2192 y S/ 4384. Mientras que en la situación actual, el $42.9 \%$ de la población percibió ingresos entre S/ 899 y S/2192, y el 14.3 \% percibió ingresos menores a S/ 899, situación que refleja un decremento del $42.9 \%$ en los ingresos promedio de la población ocupada en el subsector agricultura, explicado en gran parte por el empleo temporal que absorbe este subsector. A pesar de que la agricultura constituye un sector de abastecimiento de bienes básicos (alimentos), durante la cuarentena las actividades vinculas han sido restringidas al personal indispensable.

La situación previa a la cuarentena representó el 50.0\% de ingresos promedio entre S/ 899 y S/ 2192, mientras que el $50.0 \%$ de la población percibió ingresos mayores a S/ 4384; en tanto que en la actualidad han paralizado temporalmente sus actividades.

En el sector primario, la diferencia en la participación de los ingresos en la economía asciende a -55.6 \% evidenciándose un decremento de aproximadamente dos tercios del total; situación no vista en muchísimos años atrás, lo que pone en alerta no solo al Estado, sino al aparato productivo de este sector, para la búsqueda de alternativas de solución como la utilización intensiva de tecnologías que ayuden a su recuperación. Esta 
alternativa puede generar controversias, teniendo en cuenta que existe la alta probabilidad del desplazamiento de mano de obra. Entonces es la oportunidad de que el Estado, dentro de su manejo keynesiano, apueste por esta mano de obra desplazada y la oriente hacia su desarrollo, teniendo como prioridad el adiestramiento en el manejo de tecnología y la valoración de la creatividad.

Mientras que los efectos del COVID-19 sobre los ingresos y brechas sociales del sector secundario y sus subsectores en Tacna se ven reflejos en la siguiente Tabla.

Tabla 3. Ingreso promedio en el sector secundario

\begin{tabular}{llccccc}
\multirow{2}{*}{ Clase } & \multirow{2}{*}{ Rango } & \multicolumn{2}{c}{ Ingreso anterior } & \multicolumn{2}{c}{ Ingreso actual } & \multirow{2}{*}{ Diferencial } \\
\cline { 2 - 5 } & & Absoluto & Participación & Absoluto & Participación & \\
\hline Baja & Menor a S/ 899 & 1 & $50 \%$ & 1 & $50 \%$ & $0 \%$ \\
Media & S/ 899 - S/ 2192 & 1 & $50 \%$ & 0 & $0 \%$ & $-50 \%$ \\
\hline Total & & 2 & $100 \%$ & 1 & $50 \%$ & $-50 \%$ \\
\hline
\end{tabular}

Se deduce que el subsector manufacturero es uno de los sectores menos propensos a perder el empleo, porque no pueden ser reemplazados por una máquina, debido al componente creatividad propio del ser humano, situación que explica la estabilidad laboral en este sector, puesto que el $100.0 \%$ continúa ocupada y generando ingresos.

El subsector industria, cuyos ingresos promedios se encuentran en el rango de S/ 899 y S/ 2192, refleja su vulnerabilidad debido a que en la situación actual el $100 \%$ se encuentra paralizada o perdió el empleo.

El sector secundario evidencia un decremento del $50.0 \%$ en la participación de los ingresos en la economía, explicado en gran medida por la industria debido a que representa un sector vulnerable a la probabilidad de reemplazo de mano de obra por maquinaria, situación que plantea alternativas viables como reinventarse completamente con utilización de alta tecnología. En el caso de la manufactura, urge el apoyo estatal dentro del estado keynesiano, no solo con el apoyo de créditos para su capitalización, sino también poner a disposición de este sector económico la estructura de comercialización con la que cuenta el Estado, a través de sus embajadas promocionando dichos productos como bienes de boutique a un demandante seleccionado con capacidad de aceptar productos singulares a precios elevados.

Los efectos del COVID-19 sobre los ingresos y brechas sociales del sector terciario y sus subsectores en Tacna se evidencian en la Tabla 4:

Tabla 4. Ingreso promedio en el sector terciario

\begin{tabular}{ccccccc}
\multirow{2}{*}{ Clase } & \multirow{2}{*}{ Rango } & \multicolumn{2}{c}{ Ingreso anterior } & \multicolumn{2}{c}{ Ingreso actual } & \multirow{2}{*}{ Diferencial } \\
\cline { 3 - 6 } & & Absoluto & Participación & Absoluto & Participación & \\
\hline Baja & Menor a S/ 899 & 28 & $46.7 \%$ & 2 & $3.3 \%$ & $-43.3 \%$ \\
Media & S/ 899 - S/ 2192 & 27 & $45.0 \%$ & 6 & $10.0 \%$ & $-35.0 \%$ \\
Alta & S/ 2192 - S/ 4384 & 4 & $6.7 \%$ & 1 & $1.7 \%$ & $-5.0 \%$ \\
Más alta & S/ 4384 a más & 1 & $1.7 \%$ & 0 & $0.0 \%$ & $-1.7 \%$ \\
\hline Total & & 60 & $100 \%$ & 9 & $15.0 \%$ & $-85.0 \%$ \\
\hline
\end{tabular}

Tacna es una ciudad eminentemente comercial, situación que explica el decremento del -94.7\% de población que paralizó sus actividades o perdió su empleo. También, es preciso señalar que este sector está compuesto por la clase media y clase baja, por lo que sitúan a este subsector con mayor impacto reflejando su vulnerabilidad. Se presume que los establecimientos comerciales de venta física tienden a desaparecer, por lo que estos locales comerciales se verán en la necesidad de reinventarse a través de medios como el internet, páginas web y redes sociales, realizando el intercambio a través del delivery.

Los ingresos promedios en el subsector asesoría profesional ha disminuido su contribución a la economía en un $53.8 \%$, lo que se explica por la alta vulnerabilidad que presenta ante una situación como la estamos atravesando 
actualmente con la crisis producto de la pandemia del coronavirus.

Uno de los atractivos relacionados al turismo en nuestro país es la gastronomía; en este caso, el impacto que representa en este subsector de restaurantes es un decremento del $91.7 \%$ de su contribución a nuestra economía, lo cual resulta devastador en muchos casos para algunos restaurantes. Resultando en un cese definitivo de sus actividades, situación que requiere también reinventarse totalmente con la utilización masiva de tecnología para acceder al mercado a través de páginas web, redes sociales y el intercambio a través de delivery.

En el subsector de la actividad financiera, los ingresos promedios de la población han decrecido considerablemente en un $77.8 \%$, en su contribución a la economía, ello también explica la exposición a la alta vulnerabilidad que presenta.

Sobre el subsector música, los ingresos promedios de la población ha sido nula respecto a su contribución a la economía, evidenciándose un alto grado de vulnerabilidad, aunque existe la probabilidad de su pronta recuperación debido a su ingrediente adicional asociado a la creatividad lo que estabiliza en gran medida el riesgo de migración de la población hacia otras actividades económicas. Lo mismo ocurre con los ingresos promedios de la población en el subsector telecomunicaciones, de acuerdo a la muestra, se han extinguido debido a su alta vulnerabilidad.

En el subsector hotelería, actividad relacionada al turismo y gastronomía, el impacto del COVID-19 ha significado la desaparición de los ingresos promedios de la población debido a su alta vulnerabilidad. Asimismo, en el subsector turismo, los ingresos promedios de la población han decrecido en su totalidad respecto a su contribución a la economía, evidenciándose un alto grado de vulnerabilidad. No obstante, nuestro país posee recursos turísticos atractivos y en un futuro próximo, habiendo superado la situación de pandemia, tenderá a su recuperación económica. Por otro lado, los ingresos promedios de la población en el subsector deporte, de acuerdo a la muestra, se han extinguido comprobándose su alta vulnerabilidad.

Siendo el sector terciario o de servicios el sector más vulnerable en una situación como la que venimos atravesando debido a la emergencia sanitaria del COVID-19, evidenciándose un decremento del $85.0 \%$ de la contribución de los ingresos promedios a la economía, por lo que urge la necesidad de reinventarse completamente, orientado a la capitalización en alta tecnología. Se presume que los establecimientos comerciales de venta física tiendan a desaparecer, por lo que estos locales comerciales se verán en la necesidad de reinventarse a través de medios como el internet, páginas web, redes sociales y el intercambio a través de delivery.

Asimismo, se determinó el aporte de los ingresos de cada sector de la economía en la región de Tacna antes de la pandemia y en la actualidad, obteniendo las variaciones en los ingresos antes y durante las medidas de contingencia, según clase socioeconómica como se observa en la Figura 2.

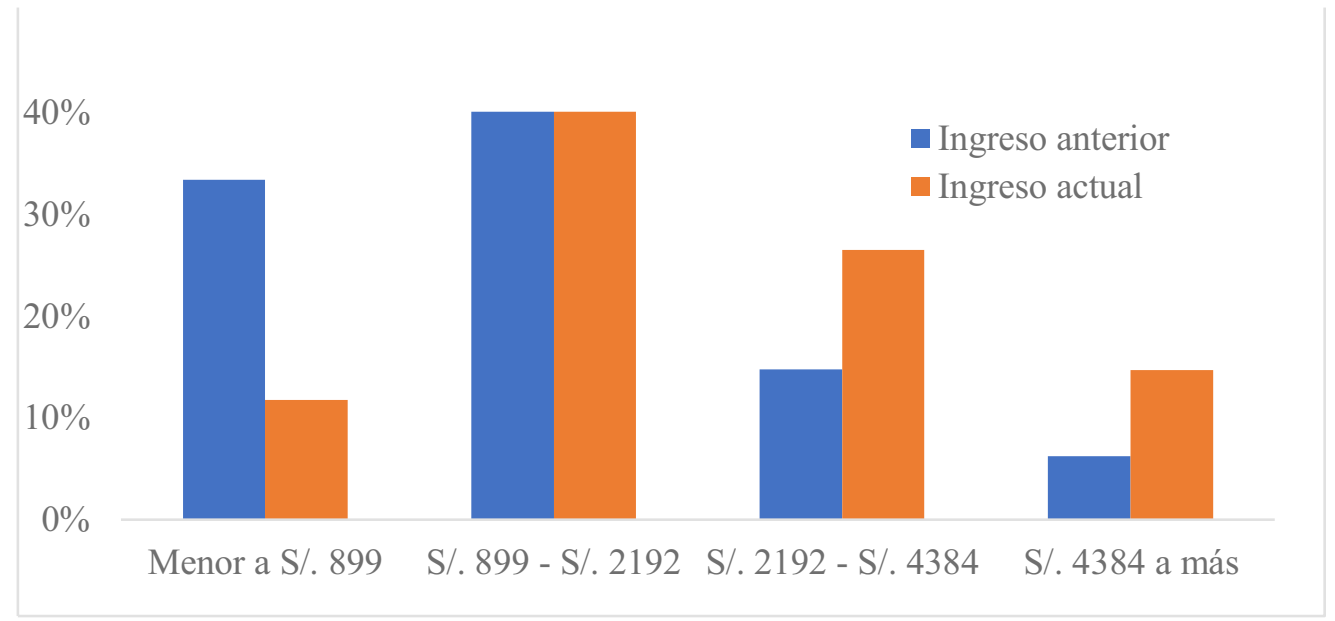

Figura 2. Comparativo de ingreso mensual antes y durante cuarentena, según clase socioeconómica

La Figura 2 muestra comparativamente que la clase alta y muy alta han incrementado sus ingresos, mientras que la población de clase media y baja ha disminuido sus ingresos o han perdido su empleo, situación que evidencia 
una polarización social teniendo en cuenta el nivel de ingresos. El COVID-19 revela que existen menos ricos con incremento significativo de sus ingresos y más pobres con el decrecimiento significativo de su ingreso. En lo que va del año, la segunda barra probablemente explica el comportamiento del ingreso del sector público, cuyo incremento después de la contingencia COVID-19 ha estado enfocado a los altos directivos, por ejemplos, rectores de universidades, poder judicial, junta nacional de justicia, entre otros.

De acuerdo con el Ministerio de Trabajo y Promoción del Empleo (2020), se explica que "los trabajadores del sector privado formal se concentraron, principalmente, en el sector servicios, abarcando a 14 mil 149 trabajadores, lo cual significó una participación del $54.0 \%$, seguido del comercio con 5 mil 246 trabajadores $(20.0 \%) "(p .5)$.

Tabla 5. Impacto del COVID-19 en el desempleo, según composición del PEA de Tacna

\begin{tabular}{|c|c|}
\hline Sectores & $\begin{array}{l}\text { PEA desempleada } \\
\text { (Durante cuarentena) }\end{array}$ \\
\hline Agrícola 1/ & $3.16 \%$ \\
\hline Minería & $2.11 \%$ \\
\hline Manufactura & $1.05 \%$ \\
\hline Construcción & $2.11 \%$ \\
\hline Comercio & $18.95 \%$ \\
\hline Servicios & $72.63 \%$ \\
\hline Total & \\
\hline
\end{tabular}

1/ Incluye ganadería, caza y silvicultura

Notamos que el impacto del COVID-19 en el desempleo, en base a la composición del PEA de Tacna, es significativo puesto que el sector servicios concentró el $72.63 \%$ de desempleo durante las medidas de contingencia por el COVID-19, seguido por el comercio con el $18.95 \%$; en tanto que el sector agrícola, minería, manufactura y construcción representan el $3.16 \%, 2.11 \%, 1.05 \%$ y $2.11 \%$ respectivamente. Los subsectores económicos que presentan mayor riesgo en perder el empleo son los servicios como el turismo, hotelería y restaurantes, asesoría profesional, actividad financiera, telecomunicaciones y comercio, situación que hace vulnerable a la población ocupada en estos sectores.

Tabla 6. Composición de la población con o sin empleo durante la cuarentena, según sector

\begin{tabular}{lcccc}
\multirow{2}{*}{ Sector } & \multicolumn{2}{c}{ Si trabajó } & \multicolumn{2}{c}{ No trabajó } \\
\cline { 2 - 5 } & Absoluto & Participación & Absoluto & Participación \\
\hline Primario & 4 & 0.286 & 5 & 0.088 \\
Secundario & 1 & 0.071 & 1 & 0.018 \\
Terciario & 9 & 0.643 & 51 & 0.895 \\
\hline Total & 14 & 1.000 & 57 & 1.000 \\
\hline
\end{tabular}

En la Tabla 6 se observa un cuadro comparativo respecto a la dinámica económica de la población impactada por el COVID-19. En el sector primario, la población que ha continuado laborando durante la cuarentena representa el $28.6 \%$, mientras que en el sector secundario representó únicamente un $7.1 \%$, y en el sector terciario el $64.3 \%$. Por otro lado, tenemos que la población que dejó de percibir ingresos económicos representa el $89.5 \%$ en el sector terciario, y mínimamente en los sectores primario y secundario con una participación del $8.8 \%$ y $1.8 \%$ respectivamente. 
Tabla 7. Test de McNemar

\begin{tabular}{|c|c|c|c|c|c|}
\hline & & & \multicolumn{2}{|c|}{$\begin{array}{l}\text { En los últimos } 03 \text { meses (abril, } \\
\text { mayo y junio) ¿Ud. ha continuado } \\
\text { laborando? }\end{array}$} & \multirow[t]{2}{*}{ Total } \\
\hline & & & No trabajó & Sí trabajó & \\
\hline \multirow{4}{*}{$\begin{array}{l}\text { Hasta antes del inicio de la } \\
\text { cuarentena ( } 15 \text { de marzo } \\
\text { de } 2020) \text {, establecido por } \\
\text { el gobierno peruano } \\
\text { debido al COVID-19 ¿Ud. } \\
\text { ha trabajado? }\end{array}$} & \multirow{2}{*}{ No trabajó } & Recuento & 68 & 0 & 68 \\
\hline & & $\%$ del total & $34.5 \%$ & $0.0 \%$ & $34.5 \%$ \\
\hline & & Recuento & 95 & 34 & 129 \\
\hline & Sí trabajó & $\%$ del total & $48.2 \%$ & $17.3 \%$ & $65.5 \%$ \\
\hline \multirow{2}{*}{ Total } & & Recuento & 163 & 34 & 197 \\
\hline & & $\%$ del total & $82.7 \%$ & $17.3 \%$ & $100.0 \%$ \\
\hline
\end{tabular}

Notamos a priori que un $34.5 \%$ de los entrevistados son personas que al inicio de la cuarentena no disponían de un empleo y que mantienen esta condición a la fecha. Al analizar el grupo de personas con empleo antes de este período de confinamiento, notamos que estas representan un $65.5 \%$ de nuestro universo de estudio, de los cuales una proporción significativa ha visto afectada esta situación al plantearse a la fecha una condición de desempleados.

El test recurre al uso de la prueba de chi cuadrado que define un valor de p de 0.000 , lo que implica que ha existido un cambio significativo en la composición de la población con trabajo en la ciudad de Tacna.

Tabla 8. Pruebas de chi-cuadrado

\begin{tabular}{ll|l} 
& Valor & $\begin{array}{l}\text { Significación } \\
\text { (bilateral) }\end{array}$ \\
\hline Prueba de McNemar & & $.000^{\mathrm{a}}$ \\
\hline $\mathrm{N}$ de casos válidos & 197 & \\
\hline
\end{tabular}

a. Distribución binomial utilizada.

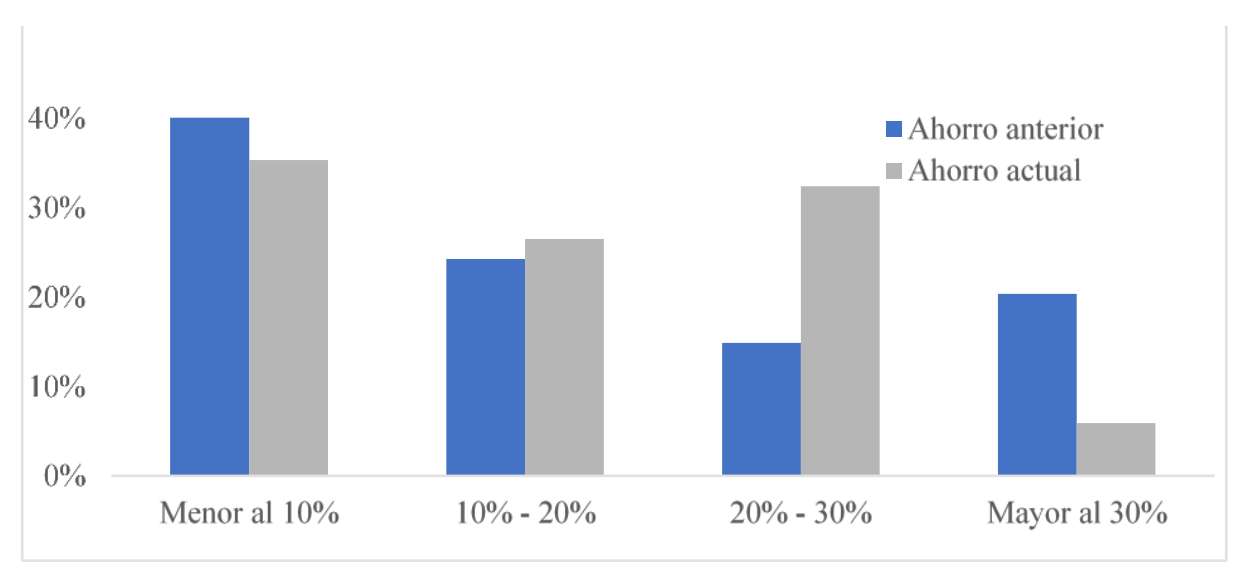

Figura 3. Comparativo de ahorro mensual antes y durante cuarentena, según clase socioeconómica.

Deducimos que solamente el $27 \%$ de los entrevistados ahorra luego de implementada las medidas de contingencia por el COVID-19. Asimismo, observamos que el porcentaje de ahorro ha disminuido, excepto en los trabajadores del sector público cuya situación laboral ha variado mínimamente, mientras que en el sector privado se observa un considerable decremento en sus ingresos que explica una disminución en la preferencia al ahorro. Los menores ingresos se traducen en menor consumo y finalmente repercute en una depresión económica que conlleva inicialmente a una recesión y, según la dinámica del flujo de dinero que el Estado gestione, existe una alta probabilidad que se desencadene una inflación. 


\section{CONCLUSIONES}

El impacto del COVID-19 en los ingresos de los trabajadores de la región de Tacna, evidencia una polarización social teniendo en cuenta el nivel de ingresos, puesto que las personas con ingresos menores a S/. 899 han perdido su empleo o paralizado sus actividades, mientras que la población de clase alta o muy alta han mantenido su nivel de ingresos porque están vinculadas principalmente al sector público.

El impacto del COVID-19 en los ingresos de los trabajadores del sector primario asciende a $-55.6 \%$ evidenciándose un decremento de aproximadamente dos tercios del total, siendo imperativa la búsqueda de alternativas como la utilización intensiva de tecnología que ayuden a su recuperación, originándose desplazamientos de mano de obra.

El impacto del COVID-19 en los ingresos de los trabajadores del sector secundario ha evidenciado una disminución del $50.0 \%$ en la participación de los ingresos, explicado en gran medida por la industria, puesto que representa un sector vulnerable a la probabilidad de reemplazo de mano de obra por maquinaria, situación que plantea alternativas viables como reinventarse completamente con utilización de alta tecnología.

El sector terciario en Tacna es el que mayor impacto ha experimentado debido al COVID-19, puesto que los ingresos promedios han decrecido en $85.0 \%$, explicado en gran parte por la paralización total del subsector comercio y debido a la vulnerabilidad de asesoría profesional, actividad financiera y telecomunicaciones ante tal circunstancia es recurrente que parte de esta PEA migre a otro sector o existe también la alternativa de reinventarse por completo en el manejo de alta tecnología.

Se ha evidenciado una disminución del $80.3 \%$ en la participación de los ingresos promedios de la PEA del sector primario, secundario y terciario en la economía, debido al COVID-19, situación que explica una disminución en el consumo y depresión económica que conlleva inicialmente a una recesión y, según la dinámica del flujo de dinero que el Estado maneje, existe la alta probabilidad que se desencadene una inflación.

En la propensión marginal al ahorro se evidencia un decremento del $73.4 \%$ debido al COVID-19 en la región de Tacna, producto de la pérdida de empleo o paralización de las actividades económicas en los sectores y subsectores de la economía.

\section{RECOMENDACIONES}

Hacer de este estudio una investigación horizontal considerando un periodo de tiempo mensual, teniendo en cuenta el contexto atípico de la economía dentro de la pandemia del COVID-19 y su evolución; con la posible consecuencia de una desaceleración de la dinámica económica no vista hasta hoy a pesar de la posibilidad de una vacuna en un futuro cercano.

\section{REFERENCIAS}

After the pandemic: Medium-term growth uncertainties. (2020). Economic Outlook, 44(2), 5-9. https://doi.org/10.1111/1468-0319.12476

Araújo Vila, N. (2020). Repercusión económica de la pandemia originada por el COVID-19 a nivel mundial. Análisis de sectores más afectados. Quipukamayoc, 28(57), 85-93. https://doi.org/10.15381/quipu.v28i57.17903

Berger, Z. D., Evans, N. G., Phelan, A. L., \& Silverman, R. D. (2020). Covid-19: Control measures must be equitable and inclusive. In The BMJ(Vol. 368). https://doi.org/10.1136/bmj.m1141

Bretas, V. P. G., \& Alon, I. (2020). The impact of COVID -19 on franchising in emerging markets: An example from Brazil. Global Business and Organizational Excellence, joe.22053. https://doi.org/10.1002/joe.22053

Campos, G. W. de S. (2020). O pesadelo macabro da Covid-19 no Brasil: entre negacionismos e desvarios. Trabalho, Educação e Saúde, 18(3), 1981-7746. https://doi.org/10.1590/1981-7746-sol00279

Chilton, S., Nielsen, J. S., \& Wildman, J. (2020). Beyond COVID-19: How the 'dismal science' can prepare us for the future. Health Economics, 29(8), 851-853. https://doi.org/10.1002/hec.4114

Clark, C. (1940). Conditions of Economic Progress. Macmillan, London. 
Evenett, S. J. (2020). Sicken thy neighbour: The initial trade policy response to COVID-19. The World Economy, 43(4), 828-839. https://doi.org/10.1111/twec.12954

Fernandez, M. (2005). Estudios Económicos - La estructura productiva en el proceso de desarrollo. http://bibliotecadigital.uns.edu.ar/scielo.php?script=sci_arttext\&pid=S2525$12952005001100001 \& \operatorname{lng}=\mathrm{en} \& \mathrm{nrm}=\mathrm{iso}$

Fisher, A. G. B. (1939). Production, primary, secondary and tertiary. Economic Record, 15(1), 24-38. https://doi.org/10.1111/j.1475-4932.1939.tb01015.x

García Acosta, V. (2005). El riesgo como construcción social y la construcción social de riesgos. SciELO, 19, $11-24$.

Instituto Nacional de Estadistica e Informática (2020). Producción nacional. Informe $N^{\circ} 07$ - Julio 2020. Lima: INEI.

IPE. (2015). Sectores Productivos | Instituto Peruano de Economía. https://www.ipe.org.pe/portal/sectoresproductivos/

Kuznets, S. (1967), "Quantitative aspects of the economic growth of nations, Economic Developmentand", Cultural Change, 15, (2).

Méndez, R., \& Prada-Trigo, J. (2014). Crisis, desempleo y vulnerabilidad en Madrid. Scripta Nova. Revista Electrónica de Geografía y Ciencias Sociales, 18(0).

Miller, S. M., Rein, M., Roby, P., \& Gross, B. M. (1967). Poverty, Inequality, and Conflict. The ANNALS of the American Academy of Political and Social Science, 373(1), 16-52. https://doi.org/10.1177/000271626737300102

Nava Bolaños, I., \& Brown Grossman, F. (2018). Determinantes del ahorro de los hogares en México: un análisis de regresión cuantílica. Economía Teoría y Práctica, 49. https://oi.org/10.24275/etypuam/ne/492018/nava

Novelo U., F. (2016). La pertinencia actual de la Teoría General de Keynes. Economía UNAM, 13(38), 41-60. https://doi.org/10.1016/j.eunam.2016.05.002

ONU. (2015). Cuentas nacionales - Análisis de los principales agregados (AMA). https://unstats.un.org/unsd/snaama/Index

Paiva, L. H., Souza, P. H. G. F. de, Bartholo, L., \& Soares, S. (2020). Evitando a pandemia da pobreza: possibilidades para o programa Bolsa Família e para o Cadastro Único em resposta à COVID-19. Revista de Administração Pública, 54(4), 1097-1110. https://doi.org/10.1590/0034-761220200243

Romero, D. E., \& Szwarcwald, C. L. (2000). Economic crisis and infant mortality in Latin America since the 1980's. Cadernos de Saúde Pública / Ministério Da Saúde, Fundação Oswaldo Cruz, Escola Nacional de Saúde Pública, 16(3), 799-814. https://doi.org/10.1590/S0102-311X2000000300028

Rosenstein-Rodan, P. N. (1943). Problems of Industrialisation of Eastern and South-Eastern Europe. The Economic Journal, 53(210/211), 202. https://doi.org/10.2307/2226317

Sen, A. (1992). Sobre conceptos y medidas de pobreza. Comercio Exterior, vol. 42 . núm 4.

Siche, R. (2020). What is the impact of COVID-19 disease on agriculture? Scientia Agropecuaria, 11(1), 3-6. https://doi.org/10.17268/sci.agropecu.2020.01.00

Souza, C. D. F. de. (2020). War economy and the COVID-19 pandemic: Inequalities in stimulus packages as an additional challenge for health systems. Revista da Sociedade Brasileira de Medicina Tropical, 53, e20200245. https://doi.org/10.1590/0037-8682-0245-2020

Timmerman, P. (1981). Vulnerability resilience and collapse of society. Toronto: Institute for Environmental Studies.

Van Nguyen, H., Van Hoang, M., Dao, A. T. M., Nguyen, H. L., Van Nguyen, T., Nguyen, P. T., Khuong, L. Q., Le, P. M., \& Gilmour, S. (2020). An adaptive model of health system organization and responses helped Vietnam to successfully halt the Covid-19 pandemic: What lessons can be learned from a RESOURCE-CONSTRAINED country. The International Journal of Health Planning and Management, hpm.3004.https://doi.org/10.1002/hpm.3004

Zimmermann, K. F., Karabulut, G., Bilgin, M. H., \& Doker, A. C. (2020). Inter-country distancing, globalisation and the coronavirus pandemic. The World Economy, 43(6), 1484-1498. https://doi.org/10.1111/twec.12969 